\title{
On Anti-Tumor Necrosis Factor-induced Systemic Lupus Erythematosus
}

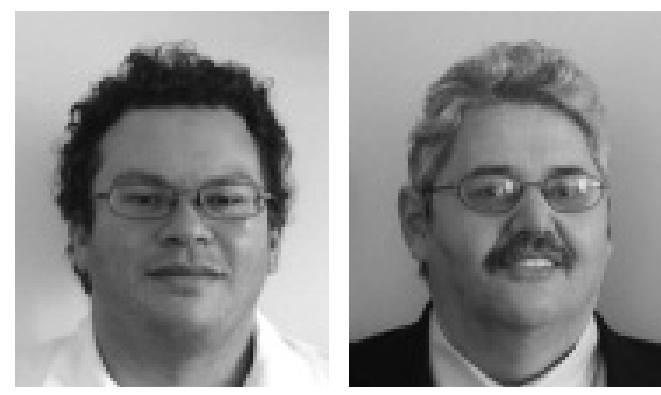

In the 1950s and 1960s Geritol tonic was heavily advertised on television as a fast cure-all if "you're feeling weak and rundown, tired or nervous." It was lampooned in a more recent sitcom as a magic elixir capable of transforming a friendless geek into the most popular kid at school. While the recent and numerous television advertisements for the anti-tumor necrosis factor (TNF) agents currently on the market do not make such outlandish claims as this, one cannot help but be awed by the overwhelming presence that these intravenously infused prescription drugs have so quickly established in the public sphere.

TNF- $\alpha$ generates a vast array of biological properties, including cellular differentiation, proliferation, and apopto$\operatorname{sis}^{1-4}$. This variability is attributed to the binding of TNF to 2 distinct transmembrane receptors that can mediate TNF-induced inflammation and cell death, the promotion of proliferative responses in $\mathrm{T}$ lymphocytes and other hematopoietic cells ${ }^{5-7}$, and the induction of apoptosis in mature activated $\mathrm{T}$ cells ${ }^{8}$. Thus, TNF can serve dually as a potent proinflammatory mediator and a key immune modulator.

Due to the multifarious effects of TNF, the results of either TNF administration or blockade have varied significantly from disease to disease and from animal models to human patients. In terms of TNF blockade, currently 3 anti-TNF agents are licensed for clinical use (the 2 monoclonal antibodies adalimumab and infliximab and a soluble TNF receptor, etanercept) and are prescribed for the treatment of rheumatoid arthritis (RA), juvenile RA, psoriatic arthritis, chronic inflammatory bowel disease (IBD), and ankylosing spondylitis 9,10 .

Contrasting the beneficial effects of TNF neutralization, TNF administration in animal models of spontaneous insulin-dependent diabetes mellitus reduces the incidence of the disease. Similarly, models of systemic lupus erythematosus (SLE) have clearly demonstrated the benefits of early administration of recombinant TNF or TNF-inducing agents on inhibition of lupus nephritis ${ }^{11-13}$. Indeed, auto- immunity and nephritis are accelerated in lupus-prone mice deficient in $\mathrm{TNF}^{14}$.

Taken together, these disparate findings suggest that the clinically relevant pathophysiological role for TNF in certain disorders is that of a proinflammatory agent. Hence, neutralization or elimination of TNF is beneficial. In other disorders, however, the role for TNF as an immune regulator seems to be more important clinically, therefore administration or induction of TNF is ameliorative (while its blockade may be detrimental).

Accordingly, the complexity of the divergent effects of TNF is further exemplified by reports that, rather than improving their condition, use of anti-TNF in patients with multiple sclerosis has resulted in immune activation and disease exacerbation ${ }^{15,16}$. Moreover, anti-TNF treatments in some patients with RA or chronic IBD incite the development of autoantibodies, neuroinflammatory disease, or SLE-like features ${ }^{17-19}$. Nevertheless, it has been suggested that anti-TNF agents can be therapeutically administered to SLE patients ${ }^{20,21}$, emphasizing the pressing need for a greater understanding of the multiple (and often divergent) roles of TNF in various conditions.

The current report by Soforo, et al in this issue of The Journal presents 6 patients who, during anti-TNF therapy, developed active SLE, fulfilling 4 or more American College of Rheumatology criteria for diagnosis ${ }^{22}$. Although lupus-like symptoms are not uncommon after TNF blockade, these cases are unique in that they are associated with major organ involvement and life-threatening manifestations. It might be difficult to argue against the possibility that some of these subjects had underlying predisposition to SLE (or undiagnosed mild SLE), and therefore, these cases represent exacerbation of SLE under anti-TNF treatment rather than induction of de novo SLE. The major point of Soforo, et al's observations, however, is that TNF blockade for SLE may be inappropriate, a view that we support regardless of whether or not these individuals represent

See Induction of SLE with TNF blockers, page 204 
genuine de novo cases of SLE, or are cases of underlying predisposition to SLE (or undiagnosed mild SLE) exacerbated by anti-TNF. The induction of SLE following TNF blockade reported in this study is further highlighted by the recent results of Jacob, et $a l^{23}$ in the lupus-prone NZM 2328 mouse model, which show that abrogating the effects of TNF by deleting both TNF receptors leads to a heightened and distinct inflammatory pathway that accelerates onset of disease, supporting the notion that anti-TNF may be contraindicated in the treatment of SLE.

Another consideration regarding these case reports is whether they represent the latest class of drug-induced lupus; or rather, represent a distinct syndrome of "anti-TNF-induced lupus." We believe that the term drug-induced SLE should remain associated with the classical presentation due to procainamide, which has a milder disease course than classical SLE, and in which anti-dsDNA antibodies generally do not develop. We tend to agree with Williams, et $a l^{24}$ that anti-TNF-induced SLE is quite distinct from classical drug-induced lupus in that it seems to be associated with a more severe disease presentation that includes cutaneous, renal, and central nervous system involvement and anti-dsDNA antibodies, and that necessitates additional treatment beyond cessation of the anti-TNF regimen. In the cases presented by Soforo, et al, discontinuation of anti-TNF agents alone was insufficient, supporting their classification as anti-TNF-induced SLE, rather than drug-induced lupus.

The significant increase in the occurrence of antinuclear antibody and anti-dsDNA antibodies with anti-TNF treatment is well documented ${ }^{25}$. However, the incidence of SLE with the use of TNF blockade is very low and the incidence of anti-dsDNA antibodies in such patients does not predict the development of full-blown SLE. Given the important immunoregulatory effects of TNF, the breakdown of which presumably can result in adverse disease manifestations, why are there not more cases/reports of systemic autoimmunity resulting from this loss of TNF-induced immune regulation? Moreover, how are we to explain some of the anecdotal reports of the benefit following anti-TNF treatment in a few patients with $\mathrm{SLE}^{20}$ ?

One potential answer is that the current anti-TNF agents provide an incomplete blockade of TNF. That is, there is still free TNF available to provide certain protective effects. Thus, some proinflammatory effects of TNF are mitigated while some of the immunoregulatory functions remain intact. A non-mutually exclusive possibility is that most patients treated so far who have benefited from anti-TNF are Caucasians possessing HLA class II genotype of DR3 and/or DR4, who have been shown to be capable of producing higher levels of TNF in response to activation ${ }^{26}$. Consequently, reducing TNF levels in such patients might mitigate the inflammatory response while preserving sufficient TNF levels to provide protective regulation. Thus, this could explain the low frequency of development of systemic autoimmunity in patients treated with anti-TNF agents. Four out of 6 patients presented by Soforo, et al are Caucasians who still developed these manifestations, but we do not know their HLA class II genotype or their TNF inducibility. However, the fact that they are Caucasian and might have DR3 or DR4 genotype, and concomitant high TNF inducibility, and still develop this adverse effect underscores the caution that must be applied with these agents.

One should also keep in mind that DR2/DQw1-positive subjects (mostly non-Caucasian) have lower TNF inducibil${ }^{i t y}{ }^{26}$, and therefore might be more prone to the harmful consequences of TNF blockade. Thus, for this population of patients even more circumspection is suggested before using TNF antagonists.

There is no question that anti-TNF agents have improved the lives of many individuals suffering from RA and are beneficial in other conditions as well. With this benefit in mind, however, TNF antagonists should not be viewed as wonder tonics or magical elixirs capable of curing all rheumatologic conditions.

\section{NOAM JACOB, MD; CHAIM O. JACOB, MD, PhD, \\ Department of Medicine, University of Southern California Keck School of Medicine, Los Angeles, California, USA}

Address correspondence to Dr. C.O. Jacob, University of Southern California, 2011 Zonal Ave., HMR 705, Los Angeles, CA 90033. E-mail:Jacob@usc.edu

\section{REFERENCES}

1. Goeddel DV, Aggarwal BB, Gray PW, Leung DW, Nedwin GE, Palladino MA, et al. Tumor necrosis factors: gene structure and biological activities. Cold Spring Harb Symp Quant Biol 1986;51:597-609.

2. Beutler B, Cerami A. Tumor necrosis, cachexia, shock, and inflammation: a common mediator. Annu Rev Biochem 1988;57:505-18.

3. Sugarman BJ, Aggarwal BB, Hass PE, Figari IS, Palladino MA, Shepard HM. Recombinant human tumor necrosis factor-alpha: effects on proliferation of normal and transformed cells in vitro. Science 1985;230:943-5.

4. Kollias G, Kontoyianni D. Role of TNF/TNFR in autoimmunity: specific TNF receptor blockade may be advantageous to anti-TNF treatments. Cytokine Growth Factor Rev 2002;13:315-21.

5. Tartaglia LA, Weber RF, Figari IS, Reynolds C, Palladino MA, Goeddel DV. The two different receptors for tumor necrosis factor mediate distinct cellular responses. Proc Natl Acad Sci USA 1991;88:9292-6.

6. Baud V, Karin M. Signal transduction by tumor necrosis factor and its relatives. Trends Cell Biol 2001;11:372-7.

7. Tartaglia LA, Goeddel DV, Reynolds C, Figari IS, Weber RF, Fendly BM, et al. Stimulation of human T-cell proliferation by specific activation of the $75-\mathrm{kDa}$ tumor necrosis factor receptor. J Immunol 1993;151:4637-41.

8. Zheng L, Fisher G, Miller RE, Peschon J, Lynch DH, Lenardo MJ. Induction of apoptosis in mature $\mathrm{T}$ cells by tumor necrosis factor. Nature 1995;377:348-51. 
9. Elliott MJ, Maini RN, Feldmann M, Kalden JR, Antoni C, Smolen JS, et al. Randomised double-blind comparison of chimeric monoclonal antibody to tumour necrosis factor alpha (cA2) versus placebo in rheumatoid arthritis. Lancet 1994;344:1105-10.

10. van Dulleman HM, van Deventer SJ, Hommes DW, Bijl HA, Jansen J, Tytgat GN, et al. Treatment of Crohn's disease with anti-tumor necrosis factor chimeric monoclonal antibody (cA2). Gastroenterology 1995;109:129-35.

11. Jacob CO, McDevitt HO. Tumor necrosis factor-alpha in autoimmune 'lupus' nephritis. Nature 1988;331:356-8.

12. Gordon C, Ranges GE, Greenspan JS, Wofsy D. Chronic therapy with recombinant tumor necrosis factor-alpha in autoimmune NZB/NZW F1 mice. Clin Immunol Immunopathol 1989; 52:421-34.

13. Ishida H, Muchamuel T, Sakaguchi S, Andrade S, Menon S, Howard M. Continuous administration of anti-interleukin 10 antibodies delays onset of autoimmunity in NZB/W F1 mice. J Exp Med 1994;179:305-10.

14. Kontoyiannis D, Kollias G. Accelerated autoimmunity and lupus nephritis in NZB mice with an engineered heterozygous deficiency in tumor necrosis factor. Eur J Immunol 2000;30:2038-47.

15. van Oosten BW, Barkhof F, Truyen L, Boringa JB, Bertelsmann $\mathrm{FW}$, von Blomberg BM, et al. Increased MRI activity and immune activation in two multiple sclerosis patients treated with the monoclonal anti-tumor necrosis factor antibody cA2. Neurology 1996;47:1531-4.

16. The Lenercept Multiple Sclerosis Study Group and The University of British Columbia MS/MRI Analysis Group. TNF neutralization in MS: results of a randomized, placebo-controlled multicenter study. Neurology 1999;53:457-65.

17. Feldmann M, Brennan FM, Maini RN. Role of cytokines in rheumatoid arthritis. Annu Rev Immunol 1996;14:397-440.
18. Sicotte NL, Voskuhl RR. Onset of multiple sclerosis associated with anti-TNF therapy. Neurology 2001;57:1885-8.

19. Sandborn WJ, Hanauer SB. Antitumor necrosis factor therapy for inflammatory bowel disease: a review of agents, pharmacology, clinical results, and safety. Inflamm Bowel Dis 1999;5:119-33.

20. Aringer M, Steiner G, Graninger WB, Höfler E, Steiner CW, Smolen JS. Effects of short-term infliximab therapy on autoantibodies in systemic lupus erythematosus. Arthritis Rheum 2007;56:274-9.

21. Aringer M, Smolen JS. The role of tumor necrosis factor-alpha in systemic lupus erythematosus. Arthritis Res Ther 2008;10:202-9.

22. Soforo E, Baumgartner M, Francis L, Allam F, Phillips PE, Perl A. Induction of systemic lupus erythematosus with tumor necrosis factor blockers. J Rheumatol 2010;37:204-5.

23. Jacob N, Yang H, Pricop L, Liu Y, Gao X, Zheng SG, et al. Accelerated pathological and clinical nephritis in systemic lupus erythematosus-prone New Zealand mixed 2328 mice doubly deficient in TNF receptor 1 and TNF receptor 2 via a Th17-associated pathway. J Immunol 2009;182:2532-41.

24. Williams EL, Gadola S, Edwards CJ. Anti-TNF-induced lupus. Rheumatology 2009;48:716-20.

25. Lin J, Ziring D, Desai S, Kim S, Wong M, Korin Y, et al. TNF alpha blockade in human diseases: an overview of efficacy and safety. Clin Immunol 2008;126:13-30.

26. Jacob CO, Fronek Z, Lewis GD, Koo M, Hansen JA, McDevitt HO. Heritable major histocompatibility complex class II-associated differences in production of tumor necrosis factor alpha: relevance to genetic predisposition to systemic lupus erythematosus. Proc Natl Acad Sci USA 1990;87:1233-7.

J Rheumatol 2010;37:3-5; doi:10.3899/jrheum.091071 